\title{
AVALIAÇÃO DA PRODUÇÃO BIOTECNOLÓGICA DE BUTANOL A PARTIR DE SORGO SACARÍNEO
}

\author{
L. J. VISIOLI ${ }^{1}$, E. A. ALVES ${ }^{2}$, A. C. M. TRINDADE ${ }^{2}$, R. C. $\mathrm{KHUN}^{2}$, M. SCHWAAB ${ }^{2}$, M. A. \\ MAZUTTI $^{2}$ \\ ${ }^{1}$ Universidade Estadual de Maringá, Departamento de Engenharia Química \\ ${ }^{2}$ Universidade Federal de Santa Maria, Departamento de Engenharia Química \\ E-mail para contato: luizjvisioli@gmail.com
}

\begin{abstract}
RESUMO - A produção de butanol a partir da fermentação da biomassa, por bactérias do gênero clostridium, é de grande importância para a oferta mundial de biocombustíveis. Nesse cenário, este trabalho buscou a avaliação da produção de biobutanol por Clostridium beijerinckii NRRL B-592 usando sorgo sacaríneo como fonte de carbono. Variáveis operacionais, como $\mathrm{pH}$ e tamanho do inóculo, e também, suplementação do meio de fermentação industrial com extrato de levedura e triptona, foram avaliadas. A concentração máxima de biobutanol obtida foi $2,12 \mathrm{~g} / \mathrm{L}$, usando $12,5 \%$ de inóculo inicial, $0,05 \mathrm{~g} / 100 \mathrm{~mL}$ de triptona, $0,1 \mathrm{~g} / 100 \mathrm{~mL}$ de extrato de levedura e com $\mathrm{pH}$ inicial ajustado em 5,5. A principal contribuição deste trabalho foi mostrar um processo sistemático para $\mathrm{o}$ desenvolvimento de um meio industrial de baixo custo a partir de sorgo sacaríneo para a produção de biobutanol.
\end{abstract}

\section{INTRODUÇÃO}

O aumento dos preços do petróleo e a necessidade de diversificar a matriz energética têm gerado o interesse na produção de combustíveis a partir de fontes renováveis em todo o mundo (Qureshi e Ezeji, 2008). A produção de etanol e butanol a partir de fermentação da biomassa tem sido bastante reportada como uma boa alternativa para a substituição do uso de combustíveis fósseis. Ainda que a produção de etanol por fermentação já esteja consolidada, principalmente no Brasil, a produção industrial do biobutanol está lentamente reaparecendo depois de ter cessado no final de anos 1960 (Qureshi et al., 2007). O butanol apresenta melhores propriedades quando comparado ao etanol, se tornando um importante candidato para utilização em substituição a gasolina, além disso, pode ser utilizado como precursor químico para a produção em massa de produtos químicos de interesse (Lu et al., 2012). Neste cenário a pesquisa sobre a produção de biobutanol tem grande relevância para otimização e redução de custo tornando possível sua aplicação em nível industrial (Mariano et al., 2010).

A fermentação anaeróbica de uma fonte de açúcares por cepa de Clostridium sp. é a principal rota biotecnológica para a produção de butanol. No entanto, esta fermentação produz também etanol e acetona, sendo então chamada de fermentação ABE (Jones e Woods, 1986). As cepas de Clostridium mais reportadas para a produção deste biocombustível são: acetobutylicum, 


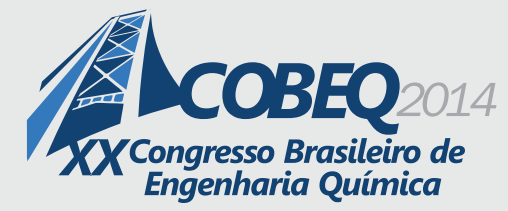

19 a 22 de outubro de 2014

Florianópolis/SC

saccharobutylicum, beijerinckii, butylicum, aurantibutyricum e tetanomorphum (Qureshi e Ezeji 2008). A manutenção da condição anaeróbia durante a fermentação é indispensável e, com isso, há um aumento no custo de operação do processo (Ezeji et al., 2013).

A viabilidade econômica da produção de biobutanol está fortemente atrelada ao custo da matéria prima utilizada na fermentação (García et al., 2011). Neste sentido, é importante a busca por um substrato barato e disponível para ser utilizado na fermentação. Resíduos são alternativa defendida por muitos autores (Qureshi e Ezeji, 2008). No entanto, o uso deste tipo de substrato tem disponibilidade questionável e muitas vezes sazonal, além de apresentarem grande variação em suas características físico-químicas. O sorgo sacaríneo pode ser uma atrativa fonte de açucares para a produção de butanol. Sorgo sacaríneo (Sorghum bicolor (L.) Moench) tem alta produção de biomassa e alta concentração de açúcares. Seu caldo contém concentração similar de glicose e sacarose e sua concentração e produtividade são altas (Yu et al., 2012).

No entanto, uma das dificuldades no uso de meios para fermentação em nível industrial é o fato da bactéria ser sensível a pequenas variações no meio de cultura. Baseado nisto, este trabalho está focado na produção de biobutanol por Clostridium beijerinckii NRRL B-592 usando caldo de sorgo sacaríneo como matéria prima. Variáveis operacionais, como $\mathrm{pH}$ e tamanho do inóculo adicionado, bem como, variáveis de suplementação do meio de fermentação industrial, como extrato de levedura e triptona, foram avaliados por meio de metodologia experimental.

\section{MATERIAIS E MÉTODOS}

\subsection{Micro-organismo, manutenção da cultura, preparação do inóculo e manipulação do micro-organismo}

O micro-organismo usado neste estudo foi Clostridium beijerinckii NRRL B-592, gentilmente fornecido por United States Department of Agriculture (USDA) - Agricultural Research Service (ARS). O micro-organismo foi transportado na forma liofilizada em vácuo. A reativação da cepa foi realizada utilizando como meio Reinforced Clostridium Medium (RCM) (Khamaiseh et al., 2012). Este procedimento durou entre 48 e 62 horas, mantendo-se a temperatura a $37^{\circ} \mathrm{C}$. A temperatura usada é aquela reportada como ótima para o crescimento celular da bactéria Clostridium beijerinckii NRRL B-592 segundo a ARS. O crescimento do micro-organismo foi realizado em tubo de ensaio selado para impedir a entrada de oxigênio.

O micro-organismo foi estocado no próprio meio RCM a temperatura entre $4-7^{\circ} \mathrm{C}$. Depois do período de estocagem, o micro-organismo cresceu novamente em meio RCM, a $37^{\circ} \mathrm{C}$, sem a necessidade de choque térmico para a reativação. O inóculo foi preparado usando $10 \%(\mathrm{v} / \mathrm{v}) \mathrm{de}$ solução semente de microorganismo. Este crescimento ocorreu em tubo de ensaio selado durante 24 horas a $37^{\circ} \mathrm{C}$ e depois foi novamente repicado nas mesmas condições. Depois de 24 horas o inóculo está pronto para a inoculação da fermentação.

O meio RCM usado nos procedimentos foi preparado usado reagentes Sigma-Aldrich ${ }^{\circledR}$. O 


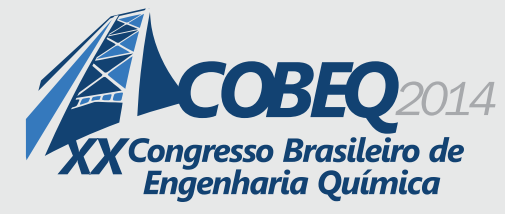

mesmo contém $(\mathrm{g} / \mathrm{L})$ : extrato de levedura $(3,0)$, extrato de carne $(10,0)$, peptona $(10,0)$, amido solúvel $(1,0)$, hidrocloreto de L-cisteina $(0,5)$, acetato de sódio $(3,0)$, agar $(0,5), \mathrm{NaCl}(5,0)$ e o $\mathrm{pH}$ foi ajustado em 6,8. Depois da preparação, o meio foi esterilizado em autoclave a $121^{\circ} \mathrm{C}$ por 20 minutos.

A manipulação necessária durante a inoculação, tanto para a preparação do inóculo, quanto para preparação para fermentação, foi feita em uma caixa anaeróbica (caixa de luvas), projetada e construída no próprio laboratório usando uma caixa comercial de polipropileno, luvas de neoprene e conexões de polivinil. Antes de iniciar a inoculação um fluxo de nitrogênio $(99,9 \%$ de pureza, fornecido pela Air Liquid $\left.{ }^{\circledR}\right)$ foi aplicado e mantido por 20 minutos para garantir da ausência de oxigênio na caixa. O meio foi borbulhado com nitrogênio para remoção de possíveis traços de oxigênio presentes no mesmo.

\subsection{Fermentação}

O processo de fermentação foi realizado usando sorgo sacaríneo como fonte de açúcar. Caldo de sorgo foi fornecido pela microdestilaria da Universidade Federal de Santa Maria, Brasil, e o sorgo foi cultivado nas coordenadas geográficas $29^{\circ} 41^{\prime} 29^{\prime \prime}$ sul, $53^{\circ} 48^{\prime} 3^{\prime \prime}$ oeste. A concentração de açúcar do caldo in natura foi determinado usando a metodologia DNS descrita por Miller (1959), e a concentração foi ajustada para $60 \mathrm{~g} / \mathrm{L}$, que é utilizada em todas as fermentações. $\mathrm{O}$ meio para a produção de biobutanol contém, além do sorgo sacaríneo como fonte de carbono, uma suplementação com $(\mathrm{g} / \mathrm{L}): \mathrm{KH}_{2} \mathrm{PO}_{4}(0,5), \mathrm{K}_{2} \mathrm{HPO}_{4} \cdot 3 \mathrm{H}_{2} \mathrm{O}(0,65)$, $\mathrm{MgSO}_{4} \cdot 7 \mathrm{H}_{2} \mathrm{O}(0,2), \mathrm{FeSO}_{4} .7 \mathrm{H}_{2} \mathrm{O}(0,01), \mathrm{MnSO}_{4} \cdot \mathrm{H}_{2} \mathrm{O}(0,01), \mathrm{NaCl}(0,01)$ e tioglicolato de sódio $(1,0)$ (Liu et al., 2010). Todos estes reagentes são da Sigma-Aldrich®. O meio ainda é suplementado com triptona e extrato de levedura, os quais são usados como variáveis do planejamento de experimentos que será melhor descrito posteriormente. Depois de preparado o meio é esterilizado em autoclave a $121^{\circ} \mathrm{C}$ por 20 minutos.

A produção de biobutanol é conduzida em fracos cônicos (erlenmeyer) de $250 \mathrm{~mL}$, contendo $100 \mathrm{~mL}$ de meio de cultura com concentração de suplementos, tamanho de inóculo e $\mathrm{pH}$ inicial definidos pelo planejamento de experimentos. A fermentação ocorre em um agitador (Tecnal ${ }^{\circledR}$ shaker, modelo TE-420) a $150 \mathrm{rpm}$ e $37^{\circ} \mathrm{C}$, durante 96 horas. O meio fermentado é centrifugado a $4000 \mathrm{rpm}$ sob temperatura de $4{ }^{\circ} \mathrm{C}$ por 15 minutos em uma centrífuga (Ependorf®, modelo 5804R). Depois disso as amostras foram estocadas a $-15^{\circ} \mathrm{C}$ para posterior análise.

Para avaliar o efeito do pH inicial, tamanho do inóculo, concentração de triptona e extrato de levedura um planejamento experimental do tipo Placket-Burman com oito experimentos nos vértices (PB8) e mais três pontos centrais foi aplicado. A Tabela 1 apresenta a amplitude das variáveis independentes investigadas. Baseado nas analises do PB8, um segundo planejamento, do mesmo tipo foi gerado para avaliar o efeito do $\mathrm{pH}$ inicial, tamanho do inóculo, concentração de triptona e extrato de levedura de acordo com a amplitude apresentada na Tabela 2. Todos os resultados foram analisados utilizando o software Statistica ${ }^{\circledR} 7.0$ (Statsoft Inc., Tulsa, OK, USA) considerando um nível de confiança de $90 \%$. 


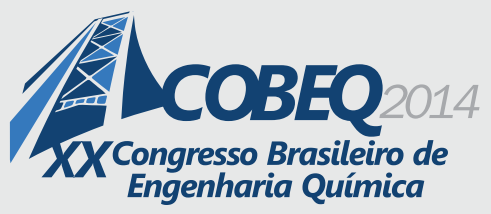

\subsection{Procedimentos analíticos}

A determinação dos solventes (acetona, butanol e etanol) foi realizada por cromatografia gasosa (Shimadzu, GC - 17A) usando uma coluna capilar DB-Wax (30m x 0,25mm x 0,25 $\mu \mathrm{m})$ com fase estacionária de polietileno glicol. A injeção foi feita em um injetor do tipo split (razão 1:20) a temperatura de $220^{\circ} \mathrm{C}$, sendo injetadas amostras de $0,2 \mu \mathrm{L}$. Foi utilizado detector de ionização de chama (FID), com temperatura mantida a $250{ }^{\circ} \mathrm{C}$. A coluna operou de acordo com a seguinte programação: $40{ }^{\circ} \mathrm{C}(8 \mathrm{~min}) ; 20^{\circ} \mathrm{C} / \mathrm{min}$ até atingir $180{ }^{\circ} \mathrm{C}$ e mantinha a esta temperatura por $10 \mathrm{~min}$. Nitrogênio foi utilizado como gás de arraste, com um fluxo de $0,8 \mathrm{~mL} / \mathrm{min}$. Metiletilcetona (MEC) foi utilizada como padrão interno da análise (Qureshi et al., 2007).

\section{RESULTADOS E DISCUSSÃO}

A Tabela 1 apresenta os resultados referentes à produção de butanol obtida no primeiro planejamento de experimentos. Como pode ser observado, a produção ocorreu somente em duas condições experimentais específicas e, mesmo assim, em baixas concentrações. No entanto, a produção ocorreu principalmente nos experimentos realizados com $\mathrm{pH}$ inicial 6,0, indicando que a amplitude empregada para esta variável específica não foi apropriada para o micro-organismo em questão. Esta suposição é confirmada analisando os efeitos de cada uma das variáveis, em forma de Diagrama de Pareto e apresentado na Figura 1. O pH apresenta um efeito negativo em relação a produção de butanol com nível de significância de $89 \%$. O fato de somente ocorrer produção do biocombustível em um valor específico de $\mathrm{pH}$ impede que se conclua que as demais variáveis não tem nenhum efeito sobre o processo, já que, estes efeitos podem ter sido ocultados pela forte dependência do $\mathrm{pH}$.

Tabela 1 - Matriz do primeiro planejamento PB8 para avaliação da influência de variáveis de processo na produção butanol

\begin{tabular}{cccccc}
\hline Experimento & $\begin{array}{c}\mathrm{pH} \\
\text { Inicial }\end{array}$ & $\begin{array}{c}\text { Inoculo } \\
(\%)\end{array}$ & $\begin{array}{c}\text { Triptona } \\
(\mathrm{g} / 100 \mathrm{~mL})\end{array}$ & $\begin{array}{c}\text { Extrato de } \\
\text { levedura } \\
(\mathrm{g} / 100 \mathrm{~mL})\end{array}$ & $\begin{array}{c}\text { Produção de } \\
\text { Butanol } \\
(\mathrm{g} / \mathrm{kg})\end{array}$ \\
\hline 1 & $8(1)$ & $5(-1)$ & $0(-1)$ & $0,4(1)$ & 0,00 \\
\hline 2 & $8(1)$ & $20(1)$ & $0(-1)$ & $0(-1)$ & 0,00 \\
\hline 3 & $8(1)$ & $20(1)$ & $0,2(1)$ & $0(-1)$ & 0,00 \\
\hline 4 & $6(-1)$ & $20(1)$ & $0,2(1)$ & $0,4(1)$ & 0,79 \\
\hline 5 & $8(1)$ & $5(-1)$ & $0,2(1)$ & $0,4(1)$ & 0,06 \\
\hline 6 & $6(-1)$ & $20(1)$ & $0(-1)$ & $0,4(1)$ & 0,00 \\
\hline 7 & $6(-1)$ & $5(-1)$ & $0,2(1)$ & $0(-1)$ & 0,17 \\
\hline 8 & $6(-1)$ & $5(-1)$ & $0(-1)$ & $0(-1)$ & 0,68 \\
\hline 9 & $7(0)$ & $12,5(0)$ & $0,1(0)$ & $0,2(0)$ & 0,00 \\
\hline 10 & $7(0)$ & $12,5(0)$ & $0,1(0)$ & $0,2(0)$ & 0,00 \\
\hline 11 & $7(0)$ & $12,5(0)$ & $0,1(0)$ & $0,2(0)$ & 0,05 \\
\hline
\end{tabular}




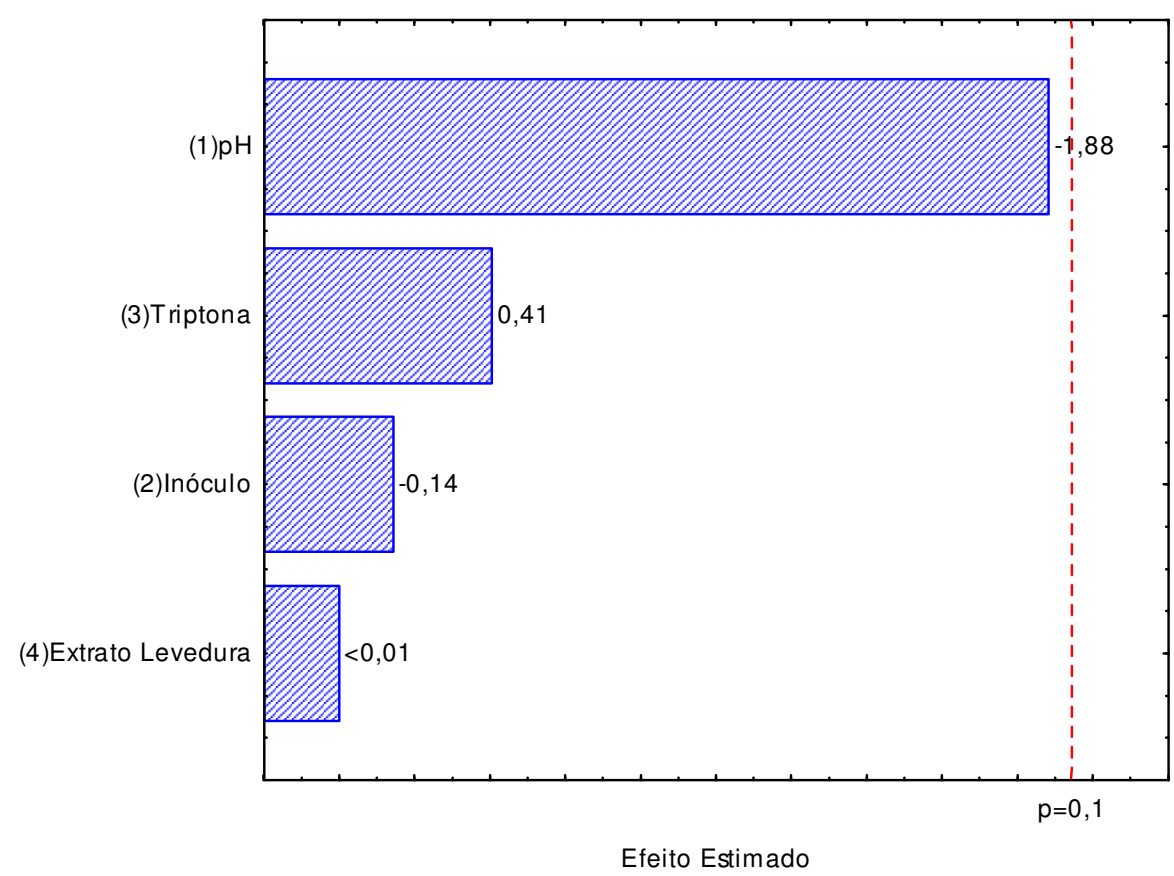

Figura 1 - Efeito das variáveis independentes do primeiro planejamento PB8 na produção de biobutanol apresentado na forma de Diagrama de Pareto.

Baseado nos resultados obtidos no primeiro planejamento de experimentos um segundo PB8 foi gerado. As variáveis investigadas foram as mesmas, mas com amplitudes alteradas. Os resultados obtidos no segundo planejamento PB8 são apresentados na Tabela 2. A maior produção de butanol foi $2,12 \pm 0,07 \mathrm{~g} / \mathrm{kg}$ e ocorreu em um dos pontos centrais do planejamento. Em outros experimentos a produção foi significativamente menor. Os dados da Tabela 2 foram usados para avaliar os efeitos principais das variáveis independentes, os quais podem ser observados na Figura 2. Como pôde ser verificado, nenhuma das variáveis investigadas foi significativa nas amplitudes estudadas. No entanto, como a condição dos pontos centrais foi aquela em que ocorreram as maiores produções, é previsível que um ajuste linear não deve ser capaz de predizer os resultados. Neste sentido, os resultados são indicativos de que as condições utilizadas nos pontos centrais do segundo planejamento PB8 são as melhores para a maximização na produção de butanol. 
Tabela 2 - Matriz do segundo planejamento PB8 para avaliação da influência de variáveis de processo na produção butanol

\begin{tabular}{cccccc}
\hline Experimento & $\begin{array}{c}\mathrm{pH} \\
\text { Inicial }\end{array}$ & $\begin{array}{c}\text { Inoculo } \\
(\%)\end{array}$ & $\begin{array}{c}\text { Triptona } \\
(\mathrm{g} / 100 \mathrm{~mL})\end{array}$ & $\begin{array}{c}\text { Extrato de } \\
\text { levedura } \\
(\mathrm{g} / 100 \mathrm{~mL})\end{array}$ & $\begin{array}{c}\text { Produção de } \\
\text { Butanol }(\mathrm{g} / \mathrm{kg})\end{array}$ \\
\hline 1 & $6(1)$ & $5(-1)$ & $0(-1)$ & $0,2(1)$ & 1,64 \\
\hline 2 & $6(1)$ & $20(1)$ & $0(-1)$ & $0(-1)$ & 0,10 \\
\hline 3 & $6(1)$ & $20(1)$ & $0,1(1)$ & $0(-1)$ & 0,28 \\
\hline 4 & $5(-1)$ & $20(1)$ & $0,1(1)$ & $0,2(1)$ & 0,00 \\
\hline 5 & $6(1)$ & $5(-1)$ & $0,1(1)$ & $0,2(1)$ & 0,67 \\
\hline 6 & $5(-1)$ & $20(1)$ & $0(-1)$ & $0,2(1)$ & 0,00 \\
\hline 7 & $5(-1)$ & $5(-1)$ & $0,1(1)$ & $0(-1)$ & 0,00 \\
\hline 8 & $5(-1)$ & $5(-1)$ & $0(-1)$ & $0(-1)$ & 0,00 \\
\hline 9 & $5,5(0)$ & $12,5(0)$ & $0,05(0)$ & $0,1(0)$ & 2,13 \\
\hline 10 & $5,5(0)$ & $12,5(0)$ & $0,05(0)$ & $0,1(0)$ & 2,18 \\
\hline 11 & $5,5(0)$ & $12,5(0)$ & $0,05(0)$ & $0,1(0)$ & 2,05 \\
\hline
\end{tabular}

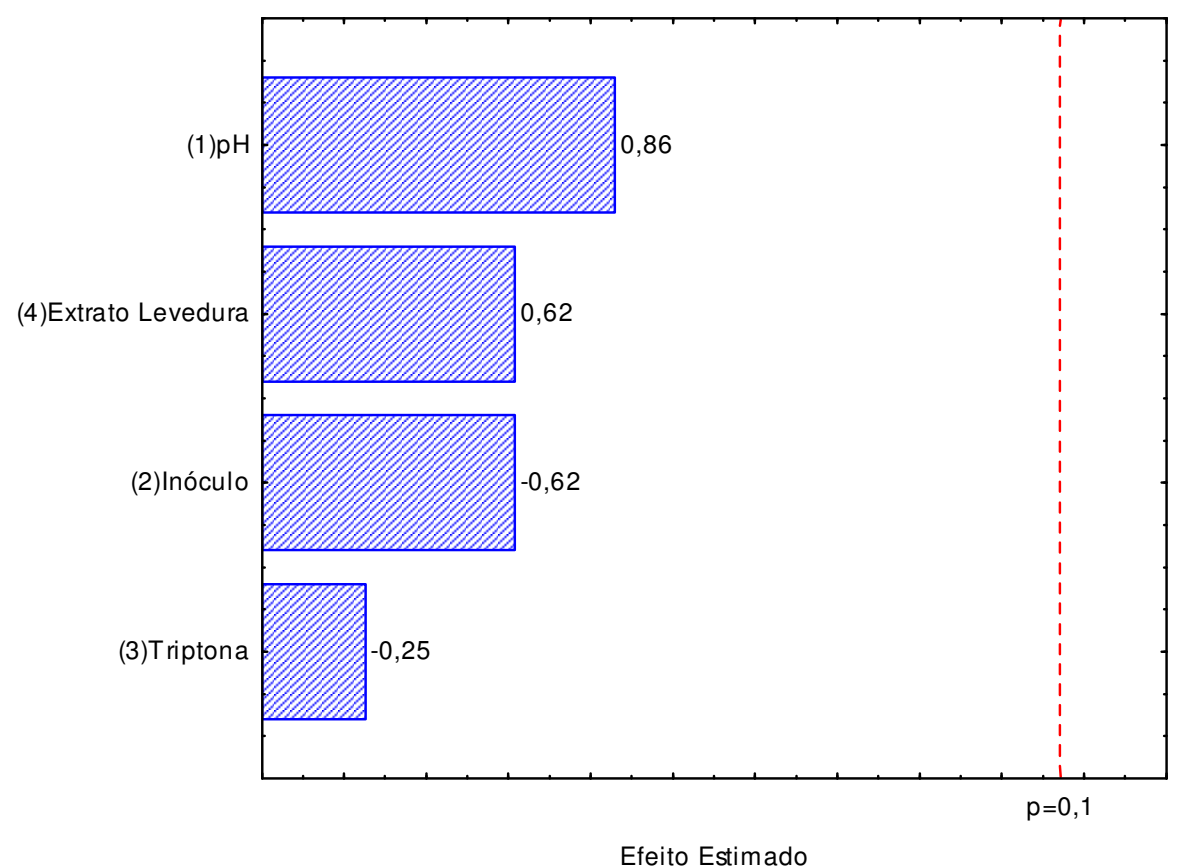

Figura 2 - Efeito das variáveis independentes do segundo planejamento PB8 na produção de biobutanol apresentado na forma de Diagrama de Pareto.

Um aspecto importante deste resultado é que a maior produção de butanol usando Clostridium beijerinckii NRRL B-592 e sorgo sacaríneo (60 g/L de açúcares redutores totais) requer baixa concentração de extrato de levedura e triptona (a metade dos valores reportados por Liu et al., 2010). 


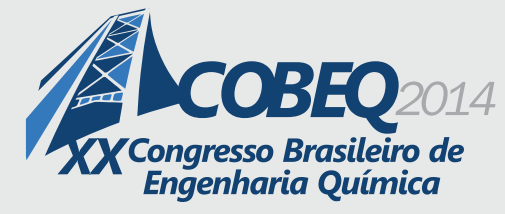

Estes reagentes têm forte impacto no custo total do processo e a redução na sua utilização pode ser importante na viabilização da produção de butanol. Este resultado demonstra que o sorgo sacaríneo pode ser utilizado em fermentações anaeróbias por clostridium.

\section{CONCLUSÕES}

Neste trabalho foi avaliada a produção de biobutanol por Clostridium beijerinckii NRRL B-592 usando caldo de sorgo sacaríneo como fonte de carbono. O máximo de butanol obtido foi $2,12 \mathrm{~g} / \mathrm{kg}$, usando tamanho de inóculo de $12,5 \%, 0,05 \mathrm{~g} / 100 \mathrm{~mL}$ de triptona, $0,1 \mathrm{~g} / 100 \mathrm{~mL}$ de extrato de levedura e pH inicial de 5,5. A principal contribuição deste trabalho foi mostrar um procedimento sistemático para o desenvolvimento de um meio industrial de baixo custo para a produção de biobutanol a partir de sorgo sacaríneo.

\section{REFERÊNCIAS}

EZEJI, T.C.; QURESHI, N.; BLASCHEK, H.P. Microbial production of a biofuel (acetonebutanol-ethanol) in a continuous bioreactor: impact of bleed and simultaneous product removal. Bioprocess Biosyst Eng, v. 36, p. 109-116, 2013.

GARCÍA, V.; PÄKKILÄ, J.; OJAMO, H.; MUURINEN, E.; KEISKI, R.L. Challenges in biobutanol production: How to improve the efficiency?. Renew Sust Energ Rev, v. 15, p. 964980, 2011.

JONES, D.T.; WOODS, D.R. Acetone-butanol fermentation revisited. Microbiol Rev, v. 50, p. 484-524, 1986.

KHAMAISEH, E.I.; KALIL, M.S.; DADA, O.; EL-SHAWABKEH, I.; YUSOFF, W.M.W. Date fruit as carbon source in RCM-modified medium to produce biobutanol by clostridium acetobutylicum NCIMB 13357. Journal of Applied Science, v.12, p. 1160-1165, 2012.

LIU, Z.; YING, Y.; LI, F.; MA, C.; XU, P. Butanol production by Clostridium beijerinckii ATCC 55025 from wheat bran. J Ind Microbiol Biotechnol, v. 37, p. 495-501, 2010.

LU, C.; ZHAO, J.; YANG, S.T.; WEI, D. Fed-batch fermentation for n-butanol production from cassava bagasse hydrolysate in a fibrous bed bioreactor with continuous gas stripping. Bioresource Technol, v. 104, p. 380-387, 2012.

MARIANO, A.P.; COSTA, C.B.B.; ANGELIS, D.F.; MAUGERI FILHO, F.; ATALA, D.I.P.; MACIEL, M.R.W.; MACIEL FLILHO, R. Dynamics of a continuous flash fermentation for butanol production. Chemical Engineering Transactions, v. 20, p. 285-290, 2010.

MILLER, G.L. Use of dinitrosalisylic acid reagent for determination of reducing sugar. Anal Chem, v. 31, p. 426-428, 1959. 
QURESHI, N.; EZEJI, T.C. Butanol, 'a superior biofuel' production from agricultural residues (renewable biomass): recent progress in technology. Biofuels, Bioprod. Bioref, v. 2, p. 319-330, 2008.

QURESHI, N.; SAHA, B.C.; COTTA, M.A. Butanol production from wheat straw hydrolysate using Clostridium beijerinckii. Bioprocess Biosyst Eng, v. 30, p. 419-427, 2007.

YU, J.; ZHANG, T.; ZHONG, J.; ZHANG, X.; TAN, T. Biorefinery of sweet sorghum stem. Biotechnol Adv, v. 30, p. 811- 816, 2012. 